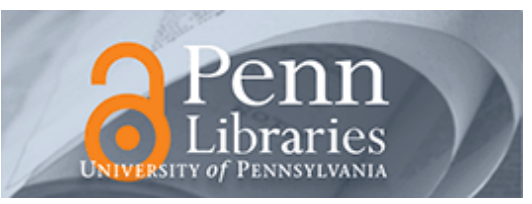

University of Pennsylvania ScholarlyCommons

$10-2015$

\title{
A Data-Driven Behavior Modeling and Analysis Framework for Diabetic Patients on Insulin Pumps
}

\author{
Sanjian Chen \\ University of Pennsylvania, sanjian@cis.upenn.edu \\ Lu Feng \\ University of Pennsylvania, lufeng@cis.upenn.edu \\ Michael R. Rickels \\ University of Pennsylvania, rickels@mail.med.upenn.edu \\ Amy Peleckis \\ University of Pennsylvania, amy.peleckis@uphs.upenn.edu \\ Oleg Sokolsky \\ University of Pennsylvania, sokolsky@cis.upenn.edu
}

See next page for additional authors

Follow this and additional works at: https://repository.upenn.edu/cis_papers

Part of the Computer Engineering Commons, Computer Sciences Commons, and the Health Information Technology Commons

\section{Recommended Citation}

Sanjian Chen, Lu Feng, Michael R. Rickels, Amy Peleckis, Oleg Sokolsky, and Insup Lee, "A Data-Driven Behavior Modeling and Analysis Framework for Diabetic Patients on Insulin Pumps", The IEEE International Conference on Healthcare Informatics 2015 (ICHI 2015) . October 2015.

The IEEE International Conference on Healthcare Informatics 2015 (ICHI 2015), Dallas, USA, October 21-23, 2015

This paper is posted at ScholarlyCommons. https://repository.upenn.edu/cis_papers/791

For more information, please contact repository@pobox.upenn.edu. 


\title{
A Data-Driven Behavior Modeling and Analysis Framework for Diabetic Patients on Insulin Pumps
}

\begin{abstract}
About $30 \%-40 \%$ of Type 1 Diabetes (T1D) patients in the United States use insulin pumps. Current insulin infusion systems require users to manually input meal carb count and approve or modify the systemsuggested meal insulin dose. Users can give correction insulin boluses at any time. Since meal carbohydrates and insulin are the two main driving forces of the glucose physiology, the user-specific eating and pump-using behavior has a great impact on the quality of glycemic control.

In this paper, we propose an "Eat, Trust, and Correct" (ETC) framework to model the T1D insulin pump users' behavior. We use machine learning techniques to analyze the user behavior from a clinical dataset that we collected on 55 T1D patients who use insulin pumps. We demonstrate the usefulness of the ETC behavior modeling framework by performing in silico experiments. To this end, we integrate the user behavior model with an individually parameterized glucose physiological model, and perform probabilistic model checking on the user-in-the-loop system. The experimental results show that switching behavior types can significantly improve a patient's glycemic control outcomes. These analysis results can boost the effectiveness of T1D patient education and peer support.
\end{abstract}

\section{Keywords}

type 1 diabetes, patient behavior, data-driven modeling and analysis, data-driven verification, blood glucose control, insulin pump, closed-loop verification, probabilistic model checking, physiological modeling, medical cyber-physical systems

Disciplines

Computer Engineering | Computer Sciences | Health Information Technology

\section{Comments}

The IEEE International Conference on Healthcare Informatics 2015 (ICHI 2015), Dallas, USA, October 21-23, 2015

\section{Author(s)}

Sanjian Chen, Lu Feng, Michael R. Rickels, Amy Peleckis, Oleg Sokolsky, and Insup Lee 


\title{
A Data-Driven Behavior Modeling and Analysis Framework for Diabetic Patients on Insulin Pumps
}

\author{
Sanjian Chen*, Lu Feng*, Michael R. Rickels ${ }^{\dagger}$, Amy Peleckis ${ }^{\dagger}$, Oleg Sokolsky*, Insup Lee* \\ * Department of Computer and Information Science, University of Pennsylvania \\ Philadelphia, PA 19104, USA \\ \{sanjian, lufeng, sokolsky, lee\}@ cis.upenn.edu \\ $\dagger$ Division of Endocrinology, Diabetes \& Metabolism \\ University of Pennsylvania Perelman School of Medicine \\ Philadelphia, PA 19104, USA \\ rickels@mail.med.upenn.edu, amy.peleckis@uphs.upenn.edu
}

\begin{abstract}
About 30\%-40\% of Type 1 Diabetes (T1D) patients in the United States use insulin pumps. Current insulin infusion systems require users to manually input meal carb count and approve or modify the system-suggested meal insulin dose. Users can give correction insulin boluses at any time. Since meal carbohydrates and insulin are the two main driving forces of the glucose physiology, the user-specific eating and pump-using behavior has a great impact on the quality of glycemic control.

In this paper, we propose an "Eat, Trust, and Correct" (ETC) framework to model the T1D insulin pump users' behavior. We use machine learning techniques to analyze the user behavior from a clinical dataset that we collected on 55 T1D patients who use insulin pumps. We demonstrate the usefulness of the ETC behavior modeling framework by performing in silico experiments. To this end, we integrate the user behavior model with an individually parameterized glucose physiological model, and perform probabilistic model checking on the user-in-the-loop system. The experimental results show that switching behavior types can significantly improve a patient's glycemic control outcomes. These analysis results can boost the effectiveness of T1D patient education and peer support.

Index Terms-Type 1 Diabetes; Patient behavior; Data-driven modeling and analysis; Data-driven verification; Blood glucose control; Insulin pump; Closed-loop verification; Probabilistic model checking; Physiological modeling; Medical Cyber-Physical Systems;
\end{abstract}

\section{INTRODUCTION}

Diabetes affects approximately 29 million people (or $9.3 \%$ of the population) in the United States and is the seventh leading cause of death [1]. Type 1 diabetics (more than 1 million in the United States) and some Type 2 diabetics depend on intensive daily insulin therapy to control their blood glucose level and avoid the numerous serious long-term complications of hyperglycemia, such as cardiovascular disease, nerve damage, blindness, and kidney damage. Advanced insulin

This research was supported in part by NSF grants CNS-1035715, IIS1231547, NIH 1U01EB012470-01, the Public Health Services research grant R01- DK091331 (to M.R.R.), the James S. McDonnell Foundation Postdoctoral Fellowship, and in part by the DGIST Research and Development Program of the Ministry of Science, ICT and Future Planning of Korea (CPS Global Center). The authors would also like to acknowledge support from the Human Metabolism Resource of the Institute for Diabetes, Obesity \& Metabolism at Penn pump technology provides continuous subcutaneous insulin infusion (CSII) therapy. It is estimated that about 400, 000 T1D patients in the United States use insulin pumps [2]. Reviews of clinical studies suggest that CSII provides improved glycemic control [3], [4].

Current insulin pumps require close supervision from the user in many operational aspects. The user needs to do a carb count for each meal so that the pump software can recommend an insulin bolus dose based on the estimated carbohydrate ratio and insulin sensitivity parameters. The user needs to approve or modify every system-suggested bolus dose as there is currently no insulin pump approved to the U.S. market that can deliver boluses automatically without user acknowledgment.

Although the use of insulin pumps has been quickly increasing in recent years, there is currently no official guidelines for medical supervision of this complicated diabetes treatment system. A recent official consensus statement by clinical expert committees stresses critical needs on evidence-based research to better understand the impact of insulin pumps on diabetic users in various physiological, psychological, and social aspects (see the AACE/ACE report [5]). Clinical studies on the use of insulin pumps predominantly focus on evaluating the impact on physiological metrics, such as the mean glucose value, rate of hypoglycemia, and HbA1c levels [6], [7], [8]. Very few results exist on understanding the behavioral aspects of how diabetic users interact with insulin pumps, which are important factors in assessing how much a patient may benefit from the CSII therapy [5]. For example, the behavioral factors include the user's eating patterns, adherence to pumprecommended insulin doses, and the level of attention to glycemic control.

Quantitative analysis of insulin pump user behavior is an important yet largely open research problem. Recent advances in insulin pump technology demonstrate a clear trend towards a high level of automation [9]. At the same time, a proven safe and effective fully closed-loop glycemic control system that requires no user supervision is not likely to be available in the near future [10]. The emerging intelligent insulin pumps introduce new challenging engineering concerns: for example, 
how much the user will trust the automation features, whether he/she will eat more carbohydrates while believing the pump's safety features can "handle it", and whether he/she may become less attentive to glucose monitoring. Patek, Chen, KeithHynes and Lee discuss the potential hazards associated with the shared human-software control in a multi-mode artificial pancreas system [11], but their work do not provide a solution to model the user behavior and quantitatively evaluate its impact on the physiology.

The fundamental heterogeneous nature of human-in-theloop control systems present critical challenges to modeling, verification, and validation. This general problem started to garner increasing attention in recent years and a few domainspecific approaches exist. Sadigh et al. propose a data-driven probabilistic modeling method to verify human driver behavior [12]. The form of the proposed model is highly specific to automotive applications and may not fit other domains. Ahmeda et al. apply generic statistical models (e.g., Bayesian Networks and Gaussian Processes) to analyzing humanautomation teamwork in unmanned aerial vehicles [13], but their method requires sufficient controlled experiments under different workload scenarios, which are usually not feasible for testing invasive clinical treatment systems. Webster et al. describe an approach to verify a home care robot system using the Brahms specification language [14], but the user models in their study capture only high-level events and cannot model the fine-grained quantitative processes such as the physiology. Because of their relative limitations, those existing techniques are not directly applicable to evaluating the physiological control systems such as the insulin pumps.

This paper makes several contributions on analyzing, modeling, and evaluating the insulin pump user behavior. First, we present statistical analysis results of a clinical dataset that demonstrate different diabetic user behavioral patterns. Second, we propose a so-called "Eat, Trust, and Correct" (ETC) modeling and analysis framework that captures the three key aspects of diabetic users' behavior in interacting with insulin pumps. We then use machine learning techniques to extract several representative user behavioral patterns from the patients' ETC models. Finally, we pair the user behavior model with an individually parameterized glucose physiological model, and evaluate how different behavioral patterns impact the individualized glucose physiology by running closedloop verification on a probabilistic model checker.

The remainder of the paper is organized as follows: Section II presents the "Eat, Trust, and Correct" (ETC) framework, the statistical analysis results of extracting ETC behavior patterns from the CSII clinical dataset, and in silico experimental results demonstrating the impact of patient behavior change on glycemic control outcomes; Section III describes the methodological details of the ETC behavior modeling, data mining, physiological model individualization, and probabilistic closed-loop verification; Finally, Section IV concludes the paper and discusses future work.

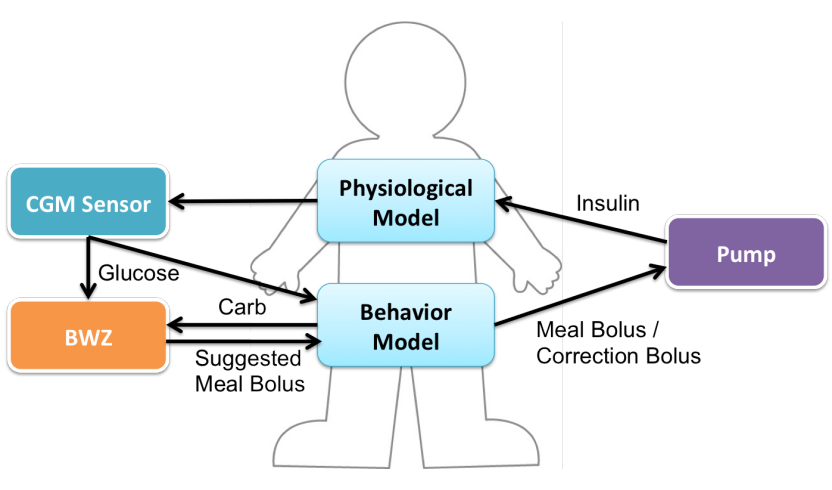

Fig. 1. The user-in-the-loop CSII system architecture.

\section{II. "EAT, Trust, AND CORRECT" (ETC) FrAMEWORK}

In this section, we propose an "Eat, Trust, and Correct" (ETC) modeling framework to classify the behavior types of T1D patients who use insulin pumps. We present the statistical analysis results of different ETC behavior patterns from a CSII clinical dataset. We also demonstrate the utility of this framework with in silico closed-loop experimental results that demonstrate the impact of patient behavior change on the glycemic control outcomes.

\section{A. The Closed-loop CSII System and the ETC Behavior Mod- eling Framework}

Figure 1 illustrates an overview of the CSII system that we consider in this paper. The system is a physiological control loop that is closed by the user. In this system, the user and patient are the same person. The Continuous Glucose Monitoring (CGM) sensor periodically transmits a subcutaneous glucose measurement to the pump and the CGM readings can also be seen by the user. There are two inputs to the patient's physiology: meal carbohydrates and insulin. When the user eats, he/she inputs the meal information into the pump Bolus Wizard $^{\circledR}$ (BWZ) feature ${ }^{1}$, which is a bolus advisory feature that calculates a recommended insulin bolus dose. The user needs to approve or modify every BWZ-recommended bolus. The user can also initiate correction insulin boluses at any time.

There is no automatic insulin delivery in current insulin pumps and the user has exclusive control authority. In this paper, we model the user behavior and evaluate how different user behavior patterns may impact the patient's glycemic control outcomes. In the closed-loop user, pump, and physiology system shown in Figure 1, the user exercises control authority through three channels: 1) eating, which represents the user's internal interaction with his/her own physiology; 2) approving or modifying BWZ-recommended boluses; 3) taking correction insulin boluses. Based on this observation, we propose the ETC behavior modeling framework that includes the three key aspects for the user's behavior in the CSII control loop:

\footnotetext{
${ }^{1}$ All patients in this study use the Medtronic systems, in which the bolus calculator is called Bolus Wizard (BWZ). In this paper, we use "BWZ" to refer to the bolus calculator in an insulin pump, but the methods and findings are certainly not specific to any particular manufacturer's products.
} 
- Eat: how often a patient eats throughout a day and what the meal carb count distributions are at different times of the day;

- Trust: the likelihood of a patient following the BWZ recommended bolus doses, and if not, how much dosage he/she adjusts;

- Correct: how often a patient takes correction boluses and what the dose distributions are at different times of the day.

\section{B. The CSII Clinical Dataset and Patient Behavior Pattern Analysis}

We collect the CSII system data from 55 T1D patients (mean \pm standard deviation of age: $45.7 \pm 15.3$ years; mean \pm standard deviation of body weight: $79.2 \pm 21.9$ kilograms) during their clinical visits to the diabetes center in the University of Pennsylvania Health System (with IRB approval). The patients all use insulin pumps augmented with a CGM sensor. The average time range of a patient's data is 30.6 days, and we assume a patient's behavior pattern does not significantly shift over this relative short time interval. Analyzing changing behavior patterns would require a larger time span of data on each patient. A national registry of T1D patients receiving care in diabetes centers, of which Penn is a participating center, indicates that $60 \%$ of adult patients use insulin pumps and $15 \%$ use CGM sensors [15]. So from the 932 patients with T1D seen at the University of Pennsylvania in the past year, 84 would be expected to use both an insulin pump and a CGM sensor. Thus, the 55 patients included in this study represent the majority of patients expected to be utilizing this sensoraugmented CSII technology in the management of their T1D at the University of Pennsylvania. ${ }^{2}$

The dataset includes two parts: 5-minute sampled CGM measurements and insulin pump data. The insulin pump data contains two sections: insulin delivery logs and BWZ data. The insulin delivery logs record the insulin basal rate at points of change, the user selected insulin bolus doses, and the pump delivered insulin bolus doses. The insulin basal rate is a low continuous infusion rate and it changes at several prescheduled times of the day. The insulin boluses consist of the mealtime boluses and non-mealtime boluses, which we call correction boluses in this paper. All data are time-stamped to the precision of second.

The BWZ calculates recommended bolus doses based on three pieces of information: (1) the meal bolus dose that is calculated from the carbohydrate input and the estimated patientspecific carbohydrate ratio, which represents the insulin dose needed for each unit carbohydrate input; (2) the correction bolus dose that is calculated from the difference between the current glucose level and the target glucose level (e.g., 100 $\mathrm{mg} / \mathrm{dL}$ ), and the estimated patient-specific insulin sensitivity, which represents the insulin dose needed for lowering a unit glucose value; (3) the active insulin on board, which is an

\footnotetext{
${ }^{2}$ We had to exclude some patients from the dataset because of missing data i.e., for those patients, there are not enough CGM measurements and insulin pump records that overlap in time.
}

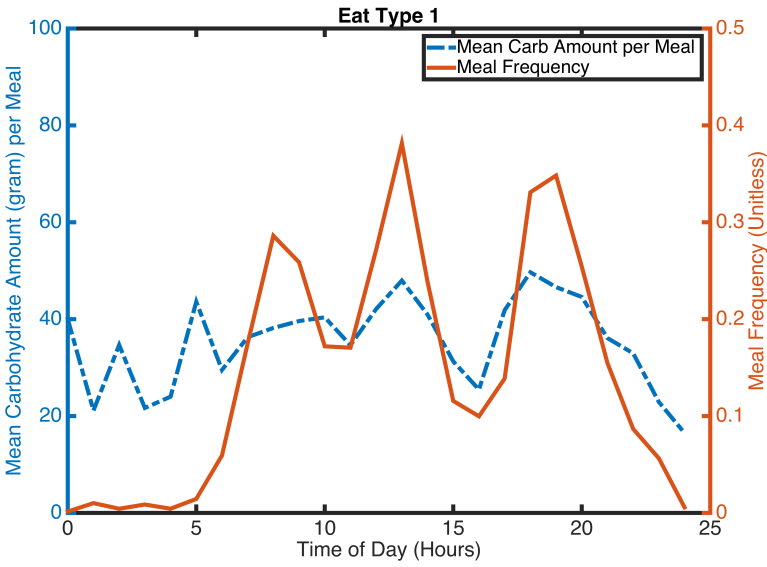

(a) Eat E1: prominent regular peak meal times with low inter-meal snacks

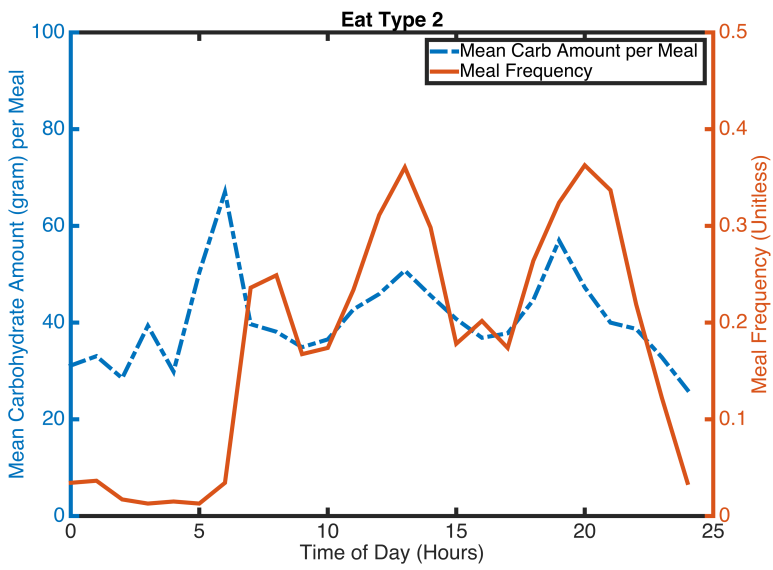

(b) Eat E2: regular peak meal times with moderate inter-meal snacks

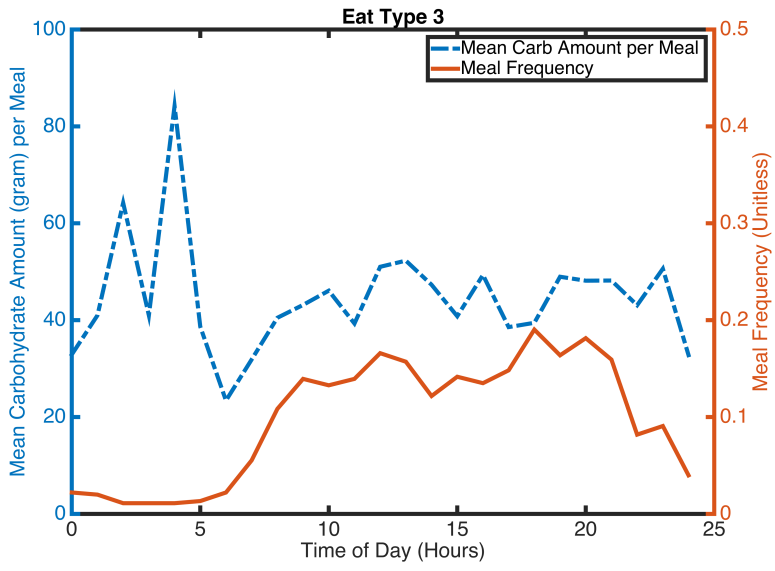

(c) Eat E3: no regular peak meal times

Fig. 2. Aggregated mean daily meal intake distributions of all patients in three Eat clusters.

estimated amount of residual insulin in the physiological system. The BWZ data section includes the following data fields: user-reported carb counts, estimated correction bolus doses, estimated meal bolus doses, estimated active insulin, the 


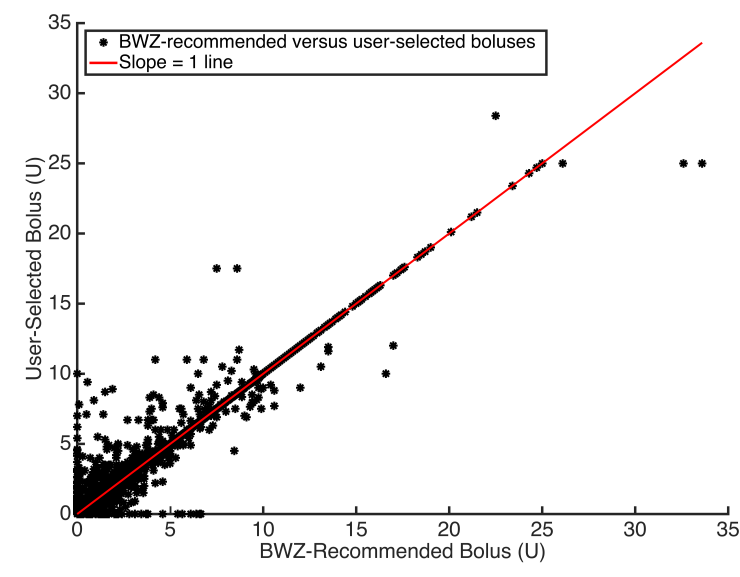

(a) Trust T1: high probability of following BWZ-recommended doses

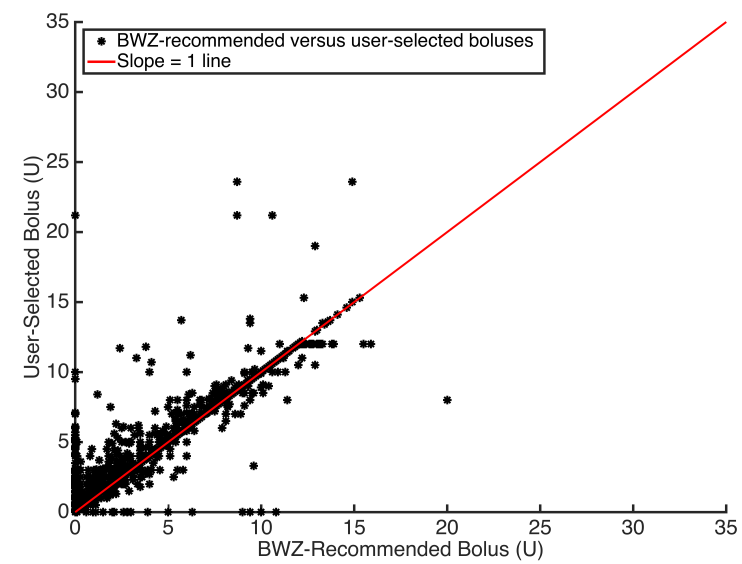

(c) Trust T3: moderate probability of increasing BWZ-recommended doses

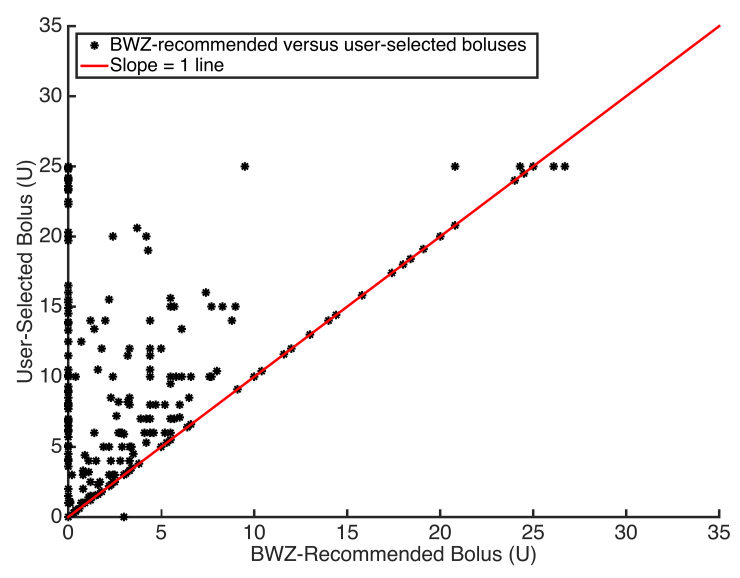

(b) Trust T2: high probability of increasing BWZ-recommended doses

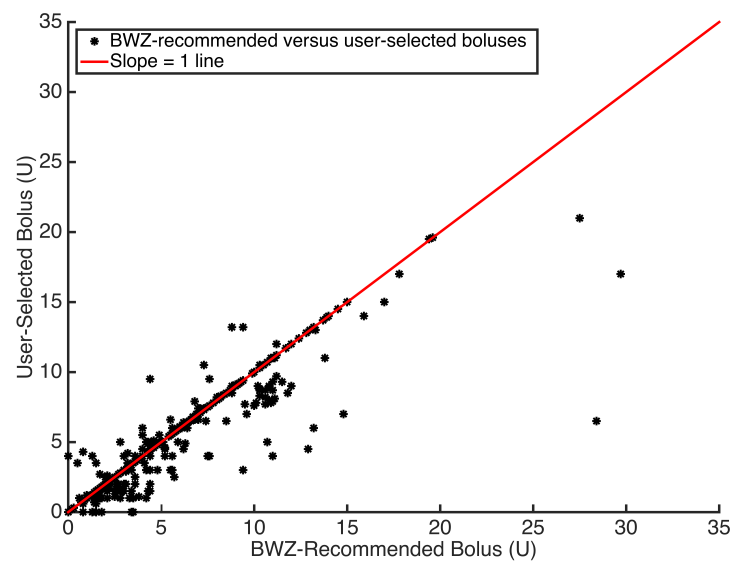

(d) Trust T4: high probability of decreasing BWZ-recommended doses

Fig. 3. Aggregated scatter plots of BWZ-recommended doses versus user-selected doses of all patients in four Trust clusters.

target glucose levels, carbohydrates ratios, insulin sensitivity values, and BWZ-recommended bolus doses. Next, we present the key findings of analyzing the ETC behavioral metrics from the CSII clinical dataset.

"Eat" Behavior Analysis. The BWZ data contains patientreported meal carb counts and meal times. For each patient, we aggregate the meal data throughout the entire data collection time interval and calculate the patient-specific distribution of the carb count at different times of the day. We then feed all patients' meal distribution data into an unsupervised learning algorithm to identify clusters (Section III explains the technical details of clustering). Each cluster contains a subset of the patients with similar meal distribution patterns. We identify three Eat clusters from the CSII dataset. Figure 2 illustrates the aggregated average daily meal intake statistics of the patients within each cluster.

To calculate meal frequency over the data collection time interval, we round each meal time to the nearest whole hour time. In Figure 2, the red solid line (mapped to the right Yaxis) is a connected-dot line of meal frequencies at 25 hour times (0-24 Hours): for example, a meal frequency of 0.3 at time 8AM in Figure 2(a) means for those patients in cluster E1, on average $30 \%$ of the days a patient would eat a meal around 8AM. The corresponding point on the blue dashed line (mapped to the left Y-axis) is the mean carb count per meal over all the meals around 8AM of all the patients within cluster E1.

Figure 2 shows three distinct daily meal intake patterns, denoted as subtypes E1, E2, and E3. The E1 subtype shown in Figure 2(a) represents patients who consistently eat three regular meals (peak frequency times are breakfast at 8AM, lunch at $1 \mathrm{PM}$, and dinner at 7PM) with some morning and afternoon snacks around 10AM and 4PM, respectively. The E1 patients rarely eat in the late night and early morning. The E2 subtype shown in Figure 2(b) represents patients who eat three regular meals with more morning and afternoon snacks than the E1 patients. The E2 patients are also more likely to eat in the late night and early morning than those in cluster E1. The average carb counts per meal at different mealtimes are similar in the E1 and E2 patients. The E3 subtype shown in Figure 2(c) 
represents patients who eat at near uniform frequencies in the daytime. Those patients do not exhibit regular peak meal times and tend to eat slightly lower carb meals when compared to the E1 and E2 patients.

"Trust" Behavior Analysis. The BWZ feature recommends a bolus dose when the user activates it at each mealtime. In the CSII dataset, we iterate through the records of userselected insulin boluses and do a timestamp matching to compare the BWZ-recommended doses with the corresponding user selected doses. For each patient, we aggregate all pairs of [BWZ-recommended dose, user-selected dose] and calculate the probabilities of the patient following, increasing, or decreasing the BWZ-recommended doses, as well as the magnitudes of dose adjustment. We then feed all patients' BWZ-adherence profiles, each of which consists of the three probabilities, into a clustering algorithm (Section III describes the details) and identify four clusters, each of which represents a group of patients with similar BWZ-adherence patterns.

Figure 3 shows the aggregated scatter plots of the [BWZrecommended dose, user-selected dose] pairs of all the patients in each cluster. The clusters are denoted as subtypes T1, T2, $\mathrm{T} 3$, and T4. The T1 subtype shown in Figure 3(a) represents patients who strongly prefer following BWZ doses and only occasionally make adjustments. Note that in Figure 3(a) and 3(c), most scatter dots are heavily overlapped on the slope $=1$ red solid line. The T2 subtype shown in Figure 3(b) represents patients who most of times prefer higher doses than the BWZ-recommended values with significant dose increases. The T3 subtype shown in Figure 3(c) represents patients who mostly follow the BWZ doses and sometimes make moderate adjustments, most of which are increasing the BWZrecommended doses. The T4 subtype shown in Figure 3(d) represents patients who mostly follow the BWZ-recommended doses and sometimes make moderate adjustments, most of which are decreasing the BWZ doses.

The clustering algorithm does not find a "negative image" subtype to T2, which would represent patients who frequently make aggressive decreasing adjustments to the BWZrecommended doses. This indicates that the BWZ dose calculation is tuned to be slightly conservative for most diabetic patients, which makes sense from a safety standpoint because insulin overdose can cause life-threatening hypoglycemia [16].

"Correct" Behavior Analysis. Unlike meal boluses, which are associated with a patient's routine daily meal pattern, both the frequency and the doses of correctional boluses highly depend on personal preference and the patient's willingness as well as availability to manage blood glucose. From the CSII dataset, we exclude the mealtime boluses from the set of all user selected boluses and calculate the aggregated distribution of correction bolus frequencies and doses over the whole hour times of a day (similar to how we treat the meal information, we round the bolus times to the nearest whole hour times). We feed the correction bolus frequency and dose distribution into a clustering algorithm (Section III describes the details) and identify three clusters of representative correction bolus

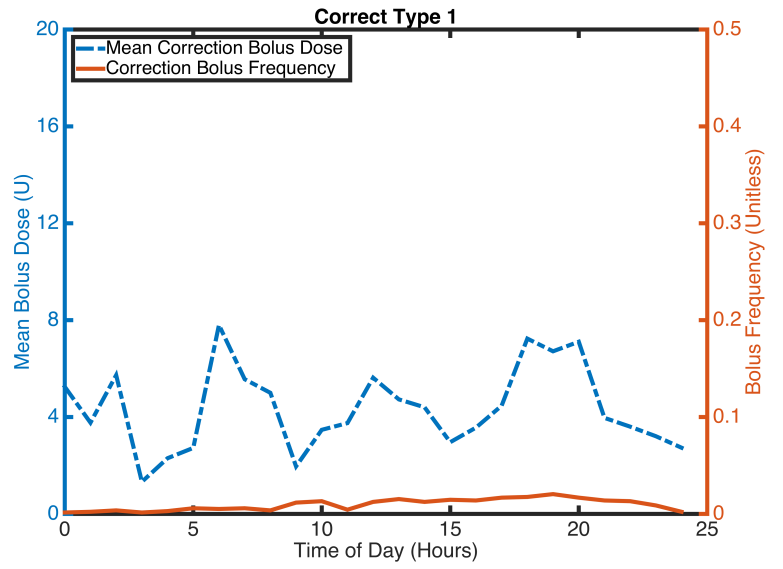

(a) Correct $\mathrm{C} 1$ : highly infrequent correction boluses

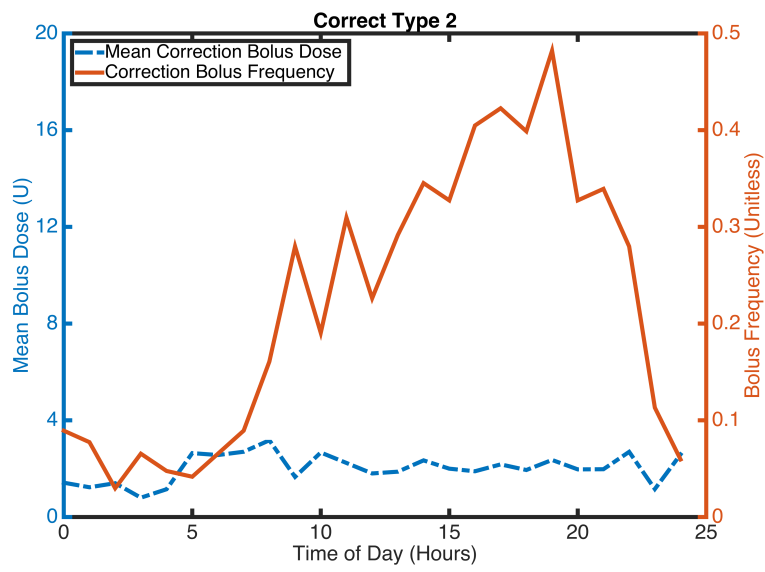

(b) Correct $\mathrm{C} 2$ : frequent correction boluses during daytime

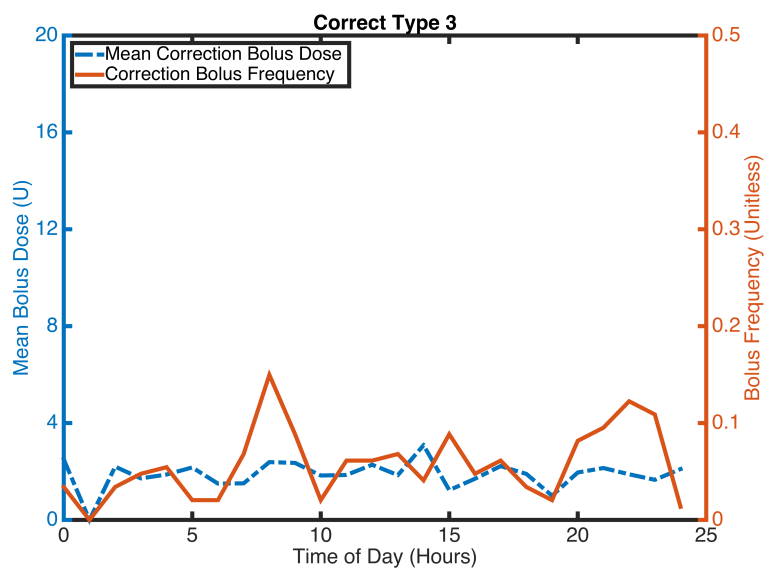

(c) Correct C3: occasional correction boluses with peaks before and after bedtime

Fig. 4. Correction bolus mean dose and frequency distributions of all patients in the four Correct clusters.

patterns, denoted as subtypes $\mathrm{C} 1, \mathrm{C} 2$, and $\mathrm{C} 3$.

Figure 4 shows the mean dose and frequency distributions of all the patients in each correction bolus cluster. Similar to 
TABLE I

FREQUENCIES OF ETC TYPES IN THE CSII DATASET.

\begin{tabular}{|c|c|}
\hline ETC Type & Frequencies (in 55 patients) \\
\hline E1T1C1 & 0.25 \\
\hline E3T1C1 & 0.16 \\
\hline E2T1C1 & 0.13 \\
\hline E2T3C1 & 0.09 \\
\hline E2T1C3 & 0.05 \\
\hline E1T3C1 & 0.05 \\
\hline E1T1C3 & 0.04 \\
\hline E3T1C2 & 0.04 \\
\hline E2T4C3 & 0.02 \\
\hline E1T3C3 & 0.02 \\
\hline E3T4C2 & 0.02 \\
\hline E3T3C2 & 0.02 \\
\hline E2T3C2 & 0.02 \\
\hline E1T2C2 & 0.02 \\
\hline E1T1C2 & 0.02 \\
\hline E3T4C1 & 0.02 \\
\hline E3T3C1 & 0.02 \\
\hline E3T2C1 & 0.02 \\
\hline
\end{tabular}

Figure 2, the frequencies (red solid lines in Figure 4) represent aggregated daily frequencies: for example, 0.1 at $8 \mathrm{AM}$ in Figure 4(a) means for all patients within cluster $\mathrm{C} 1$, on average $10 \%$ of the days a patient would take a correction bolus around 8AM. The dashed dot blue line in the same figure represents the corresponding mean dose distribution over all the patients in the cluster.

The subtype $\mathrm{C} 1$ shown in Figure 4(a) represents patients who rarely take any correction boluses. The subtype $\mathrm{C} 2$ shown in Figure 4(b) represents patients who frequently take correction boluses during the daytime. The subtype $\mathrm{C} 3$ shown in Figure 4(c) represents patients who take correction boluses at a moderate frequency throughout the day, with two peak times around 8AM and 10PM, i.e., before and after the typical bedtime. The dose distributions in all three subtypes are similar: the correction bolus doses are mostly in the midlow range $(0-5 \mathrm{U})$, which is consistent with common clinical guidelines of diabetes self-management: high dose boluses at non-meal times are usually not recommended as they may cause hypoglycemia.

Summary and Remarks of the ETC Behavior Analysis. The CSII dataset includes T1D patients who visit the clinic during the data collection period starting in May 2014. We include a patient's data as long as the time ranges of the insulin data and CGM data at least partially overlap, because we need time-matched insulin and glucose data to individualize the physiological model for closed-loop evaluation. We do not have any other patient screening criteria for data inclusion. The current CSII dataset has 55 T1D patients whose ages range from 23 to 79 and body weights range from $50 \mathrm{~kg}$ to $175 \mathrm{~kg}$. As noted before, the set of patients represents the majority of T1D patients at the study site who use insulin pumps.

The three Eat subtypes, four Trust subtypes, and three Correct subtypes generate 36 possible ETC combinatorial types. Table I lists the frequencies of the ETC types observed in the CSII dataset (the remaining ETC types not presented in
TABLE II

THE EFFECT OF BEHAVIOR (ETC TYPES) CHANGE ON THE HYPOGLYCEMIA AND HYPERGLYCEMIA RATES FOR A PATIENT WITH A HIGH BASELINE HYPOGLYCEMIA RATE

\begin{tabular}{|c|c|c|c|}
\hline & ETC Type & $\begin{array}{c}\text { Hypoglycemia } \\
\text { Rate (\%) }\end{array}$ & $\begin{array}{c}\text { Hyperglycemia } \\
\text { Rate (\%) }\end{array}$ \\
\hline Actual type & E3T2C1 & $\mathbf{6 . 9 3}$ & 8.43 \\
\hline Change & E1T2C1 & 6.20 & 12.78 \\
E subtype & E2T2C1 & 5.99 & 13.72 \\
\hline Change & E3T1C1 & 0.02 & 10.33 \\
T subtype & E3T3C1 & 0.04 & 10.09 \\
& E3T4C1 & 0.02 & 11.05 \\
\hline Change & E3T2C2 & 7.04 & 6.30 \\
C subtype & E3T2C3 & 6.95 & 7.93 \\
\hline Change & E2T1C1 & 0.04 & 16.46 \\
multi-subtypes & E2T2C1 & 5.99 & 13.72 \\
& E3T1C3 & 0.10 & 9.76 \\
& E2T1C3 & 0.08 & 15.42 \\
\hline
\end{tabular}

the table are never observed on any of the patients in the CSII dataset). The most frequent Trust and Correct combination is $\mathrm{T} 1 \mathrm{C} 1$ (54\% of patients are in this subtype), indicating that a significant portion of patients rarely make adjustments to the BWZ recommended doses or take correction boluses. The T3 subtype is less common than T1 but still represents $22 \%$ of patients. The $\mathrm{T} 2$ and $\mathrm{T} 4$ subtypes represent uncommon subtypes, but they are so distinct from $\mathrm{T} 1$ and $\mathrm{T} 3$, and some patients do exhibit those Trust patterns. $\mathrm{C} 2$ and $\mathrm{C} 3$ are less common subtypes but do cover $27 \%$ of the patients. The three Eat subtypes all cover a significant subset of the patients, indicating diverse eating habits across the insulin pump user population.

The different meal intake, BWZ-adherence, and correction bolus patterns could clearly have distinct impact on the glucose physiology. Our clinician collaborators who work in diabetes treatment and advanced research review the analysis results and think that the ETC data mining approach extracts new information that is highly clinically relevant but is not part of the current standard pump software downloads, which typically only include overall statistics such as the means and variances of CGM values. The clinicians believe the ETC metrics provide important insights for caregivers to better understand how each patient's own glucose-related behavior impacts his/her glucose levels, which would ultimately promote the efficacy of treatment and enable better glycemic control.

\section{Effects of ETC Type Change on the Glycemic Control Outcomes}

We demonstrate the utility of our patient behavior modeling framework with in silico experiments to illustrate the impact of patient behavior (ETC type) change on the glucose control outcomes. Such analysis results can inform T1D patient education and peer support [17].

We evaluate the glucose control outcomes using the hypoglycemia and hyperglycemia rates: that is, the percentage of glucose readings below $70 \mathrm{mg} / \mathrm{dL}$ (hypoglycemia [18]) or above $180 \mathrm{mg} / \mathrm{dL}$ (hyperglycemia [18]) over a certain period 
TABLE III

THE EFFECT OF BEHAVIOR (ETC TYPE) CHANGE ON THE HYPOGLYCEMIA AND HYPERGLYCEMIA RATES FOR A PATIENT WITH A HIGH BASELINE HYPERGLYCEMIA RATE

\begin{tabular}{|c|c|c|c|}
\hline & ETC Type & $\begin{array}{c}\text { Hypoglycemia } \\
\text { Rate }(\%)\end{array}$ & $\begin{array}{c}\text { Hyperglycemia } \\
\text { Rate }(\%)\end{array}$ \\
\hline Actual type & E1T1C1 & 0 & $\mathbf{4 3 . 9 2}$ \\
\hline Change & E2T1C1 & 0 & 44.38 \\
E subtype & E3T1C1 & 0 & 41.62 \\
\hline Change & E1T2C1 & 0 & 39.13 \\
T subtype & E1T3C1 & 0 & 43.46 \\
& E1T4C1 & 0 & 45.31 \\
\hline Change & E1T1C2 & 0 & 41.59 \\
C subtype & E1T1C3 & 0 & 43.47 \\
\hline & E1T2C2 & 0 & 37.22 \\
Change & E3T2C1 & 0 & 35.45 \\
multi-subtypes & E3T1C2 & 0 & 38.01 \\
& E3T2C2 & 0 & 32.56 \\
\hline
\end{tabular}

of time. Section III describes the details of the in silico closedloop experiments. Here, we highlight the experimental results by showing the impact of ETC type change on the hypoglycemia and hyperglycemia rates for two sample patients.

Table II presents the results for a patient with a high baseline hypoglycemia rate $(6.93 \%)$ in the experiments. The patient's actual behavior type is E3T2C1. As illustrated in Table II, by changing the E subtype only, the hypoglycemia rate only drops slightly. By changing the $\mathrm{T}$ subtype, the patient's hypoglycemia rate would significantly decrease. The patient has a high likelihood of increasing the BWZ dose (the actual subtype is T2). The results in Table II suggest that if the patient follows the BWZ dose (T1) or even gives smaller doses (T4), the expected hypoglycemia rate would drop to around $0.02 \%$, which would be a beneficial outcome. The fundamental challenge in glycemic control is that reducing the correction doses can mitigate hypoglycemia, but at the same time, it would also put the patient at a higher risk of hyperglycemia: for example, if the patient behaves as E3T1C1, then the expected hyperglycemia rate would be around $10.33 \%$, which is slightly higher than the value of $8.43 \%$ with the actual type E3T2C1. Optimizing the CSII dose always comes down to balancing the risk between hypoglycemia and hyperglycemia. Hypoglycemia is a more critical short-term safety concern: extreme hypoglycemia is life-threatening. Furthermore, the current population baseline hyperglycemia rate among Type 1 diabetics is in the high range of $20 \%$ to $40 \%$ [18]. Therefore, significantly reducing hypoglycemia at the cost of slightly increasing hyperglycemia is justifiably beneficial to the patient. The results also show that changing the $\mathrm{C}$ subtype would not reduce the hypoglycemia rate for this patient. These experiment results could inform patient education: for example, clinicians may consider suggesting this patient to follow the BWZ-recommended doses rather than frequently selecting higher doses.

Table III presents the results for another patient with a high hyperglycemia rate $(43.92 \%)$ in the experiments. The patient's actual behavior type is E1T1C1 and does not experience hypoglycemia. Based on the results, in order to reduce the

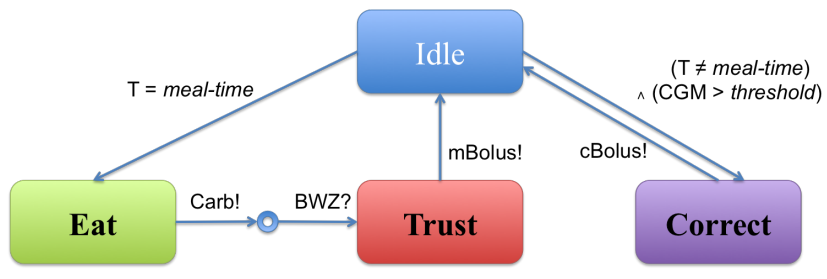

Fig. 5. An Overview of the user behavior.

hyperglycemia rate, we would suggest the patient to reduce carbohydrate intake. For example, by changing the E subtype from E1 to E3, the expected hyperglycemia rate drops to around $41.62 \%$. In addition, the treatment outcomes would be further improved if the patient gives higher BWZ dose (T2) or take more correction boluses (C2), as highlighted in Figure III. The optimal treatment outcomes can be achieved if the patient changes behavior in all three aspects of the ETC types: the expected hyperglycemia rate drops to $32.56 \%$ if the patient acts as type E3T2C2, which would be a significant improvement compared to the patient's baseline hyperglycemia rate.

\section{METHODS}

In this section, we first present our approach of modeling the patient behavior and identifying representative clusters of the individual patient behavior patterns using unsupervised learning techniques. Then we describe how we individualize an existing generic physiological model to the CSII dataset. Finally, we integrate the behavior model and physiological model and run in silico experiments via closed-loop probabilistic model checking to evaluate how changing the user behavior may impact the glucose outcomes.

\section{A. Patient Behavior Modeling and Clustering}

Figure 5 illustrates the operational workflow in the use of the CSII system. At non-meal time, the patient interacts with the system by requesting correctional boluses. At mealtimes, the patient inputs the carb count and take meal-time boluses with the assistance of the BWZ feature. We identify three main user behavior factors and represent each patient's behavior profile as an ETC type. Next, we describe how to quantitatively model the ETC types and cluster the patients' behavior models.

Quantitative Modeling of ETC Behavior Types. Eat and Correct are time-sensitive metrics because the meal carbohydrate intake and correctional boluses both greatly depend on the time of the day. To incorporate both the time of the day and the magnitude information of the meal carbohydrate intake, we partition the time of the day into $N_{E}$ time intervals $T_{1}^{E} \ldots T_{N_{E}}^{E}$, where $\cup_{i=1}^{N_{E}} T_{i}^{E}=[0,24]$, and we partition the possible value range of a carb count into $M_{E}$ intervals $S_{1}^{E} \ldots S_{M_{E}}^{E}$, where $\cup_{i=1}^{M_{E}} S_{i}^{E}=\mathbb{R}^{+}$. Similarly, for the correction bolus, we partition the time of the day into $N_{C}$ intervals and partition the possible value range of a bolus dose into $M_{C}$ intervals.

Let $X_{E}$ denote the Eat matrix of dimension $N_{E}$ by $M_{E}$ and let $X_{C}$ denote the Correct matrix of dimension $N_{C}$ by 
TABLE IV

FOUR OUTPUT CENTROIDS RETURNED BY THE K-MEANS ALGORITHM RUNNING OVER 55 PATIENTS' TRUST PROBABILITY DISTRIBUTIONS $D_{T}$

\begin{tabular}{|c|c|c|c|}
\hline Centroid ID & $P_{H}$ & $P_{F}$ & $P_{L}$ \\
\hline 1 & 0.05 & 0.92 & 0.03 \\
\hline 2 & 0.73 & 0.26 & 0.01 \\
\hline 3 & 0.21 & 0.75 & 0.04 \\
\hline 4 & 0.07 & 0.63 & 0.30 \\
\hline
\end{tabular}

$M_{C}$. An element $x_{i j}$ in $X_{E}$ represents the probability of the patient eating a meal with the carb count in the interval $S_{j}^{E}$ within the time interval $T_{i}^{E}$. Similarly, an element $x_{i j}$ in $X_{C}$ represents the probability of the patient taking a correction bolus with dose in the interval $S_{j}^{C}$ within the time interval $T_{i}^{C}$. Clearly, for each row, we have $\forall i \sum_{j} x_{i j}=1$. We estimate the probability matrices $X_{E}$ and $X_{C}$ for each patient from the CSII dataset by assigning the meal carb counts and boluses into the corresponding value intervals and time intervals, and then counting the frequencies.

Trust mainly indicates a patient's BWZ-adherence level and is less sensitive to time of the day. ${ }^{3}$ We aggregate all the [BWZ recommended dose, user selected dose] pairs of each patient and estimate the probabilities of the patient increasing, following, or decreasing the BWZ-recommended doses: those three probabilities are denoted as $P_{H}, P_{F}$ and $P_{L}$, respectively. Let $D_{T}$ denote the probability distribution $D_{T}=\left\langle P_{L}, P_{F}, P_{H}\right\rangle$.

Finally, the $k$-th patient's complete ETC model is a tuple $E T C_{k}=\left\langle X_{E}, X_{C}, D_{T}\right\rangle$.

Clustering of ETC Models. To goal of clustering is to identify common patterns within the $E T C_{k}$ tuples estimated on the CSII dataset. Note that $E T C_{k}$ contains $N_{E} \times\left(M_{E}-1\right)+$ $N_{C} \times\left(M_{C}-1\right)+2$ free dimensions, which can quickly out-grow the size of the dataset $(n=55)$. Clustering highdimensional data introduces a number of computational and theoretical challenges, as noted in extensive machine learning research such as [19]: for example, the dimensions become hard to think of and visualize, computational intractability (the curse of dimensionality), and the relative distance between samples converges as dimensions grow. Numerous machine learning techniques exist on tackling this challenge [20]. In the CSII dataset, not only that the number of free variables can be far greater than the sample size, the clinical meanings of the variables are highly heterogeneous and the three elements in $E T C_{k}$ are estimated from disjointed subsets of the data. Therefore, we decompose the clustering problem into three parallel sub-problems: clustering each of $X_{E}, X_{C}$, and $D_{T}$ independently. The ETC clusters would consist of combinations of the sub-clusters.

We run the k-means clustering algorithm [21] on the 55 Trust probability distributions $D_{T}$ 's. After experimenting different numbers of cluster $K$ and the initialization settings,

\footnotetext{
${ }^{3}$ It is possible that the BWZ-adherence level may exhibit short-term fluctuations, e.g., a patient may be more likely to simply follow BWZ doses when he/she is busy with other things; examining short-term Trust patterns is part of our future work.
}

TABLE V

COMPARISON OF POPULATION-WIDE GLUCOSE STATISTICS OF THE CSII DATASET AND THE MODEL-SIMULATED GLUCOSE DATA GIVEN THE SAME INSULIN \& MEAL INPUTS. BG DENOTES THE BLOOD GLUCOSE LEVEL. ALL BG OUTCOMES ARE IN THE UNIT OF MG/DL.

\begin{tabular}{|c|c|c|}
\hline & $\begin{array}{c}\text { CSII } \\
\text { Dataset BG }\end{array}$ & $\begin{array}{c}\text { Model } \\
\text { Simulated BG }\end{array}$ \\
\hline Mean BG & 163 & 159 \\
\hline Max BG & 365 & 379 \\
\hline Min BG & 50 & 49 \\
\hline BG $>180$ & $35 \%$ & $30 \%$ \\
\hline BG $<70$ & $3 \%$ & $3 \%$ \\
\hline BG in $[70,180]$ & $62 \%$ & $67 \%$ \\
\hline
\end{tabular}

we choose $K=4$ considering both the distribution of pointto-centroid distances and the practical implications: the output cluster centroids must have clear interpretations that are easily understandable by clinicians. The four output centroids are listed in Table IV. The four centroids correspond to the four Trust types T1 - T4 presented in Figure 3.

The Eat and Correct matrices $X_{E}$ and $X_{C}$ are each defined by two types of partitions: the time partitions and the magnitude partitions. Choosing the numbers of partitions $N_{E}, M_{E}$, $N_{C}$, and $M_{C}$ involves a trade-off: on one hand, the partitions must distinguish different meal carb counts and bolus doses at a reasonably granularity; on the other hand, too fine-grained partitions cause data sparseness.

In this paper, we empirically choose $N_{E}=6, M_{E}=8$, $N_{C}=6$, and $M_{C}=11$, which are consistent with common clinical knowledge: patients typically take six or less meals a day, $>200$ grams is a very high-carb meal, and $10 \mathrm{U}$ is a common maximum dose limit on the pumps for safety. ${ }^{4}$ We use the same time of the day partition for both $X_{E}$ and $X_{C}$ : the six time cut-off points are 7AM, 10AM, 1PM, 4PM, 7PM, and 10PM. The six time intervals represent breakfast, morning snack, lunch, afternoon snack, dinner, and evening snack times. We partition the meal carb count into eight intervals: the cut-off points are $[0,15,30,40,50,75,100,200]$ grams; the rationale is that by analyzing all meal carb counts in the CSII dataset, we observe that most meal carb counts are in the range of $0-75$ grams and therefore we assign denser intervals in that range. The correction insulin bolus dose is partitioned into eleven intervals: the cut-off points are $0-10$ $\mathrm{U}$ with a step-size of $1 \mathrm{U}$; the rationale is that most insulin boluses in the CSII dataset are within the 0-10 $\mathrm{U}$ range and clinicians consider $10 \mathrm{U}$ to be a very high bolus dose (some clinical insulin protocols set $10 \mathrm{U}$ as the maximum limit of a bolus [22]).

Under the above partition scheme, $X_{E}$ is a $6 \times 8$ matrix and $X_{C}$ is a $6 \times 11$ matrix, which still include too many features for 55 samples. We apply a two-round clustering heuristic ${ }^{5}$ : to cluster the $X_{E}$, in the first round, we run k-means algorithm to identify clusters of meal carb count distributions

\footnotetext{
${ }^{4}$ In our future work, we will systematically investigate the performance of the clustering algorithm under different parameter settings.

${ }^{5} \mathrm{We}$ will evaluate alternative methods of tackling the dimensionality challenge in our future work.
} 
TABLE VI

STATISTICS OF THE PER-PATIENT DIFFERENCES BETWEEN THE CSII GLUCOSE MEASUREMENTS AND THE MODEL-SIMULATED GLUCOSE VALUES. BG DENOTES THE BLOOD GLUCOSE LEVEL.

\begin{tabular}{|c|c|}
\hline Metric & Value \\
\hline Mean Difference of Per-Patient Mean BG & $14 \mathrm{mg} / \mathrm{dL}$ \\
\hline Mean Difference of Per-Patient BG > 180 Percentage & $5 \%$ \\
\hline Mean Difference of Per-Patient BG < 70 Percentage & $1 \%$ \\
\hline Mean Difference of Per-Patient BG in [70,180] Percentage & $6 \%$ \\
\hline
\end{tabular}

at each time interval, i.e., on each row of $X_{E}$; the cluster ID output of each row in $X_{E}$ from the first round is the input to the second round of clustering, where we identify clusters of meal-over-time distributions. For example, the first row of $X_{E}$ represents the carb count distribution at the breakfast time. We collect the breakfast carb count distributions of 55 patients, each of which contains 8 probabilities, and we run k-means clustering to identify $K$ breakfast clusters. We repeat this for all 6 rows of $X_{E}$. At the end of the first round of clustering, each patient's $X_{E}$ is mapped to a six-dimension cluster membership $K C=\left[K_{1} \ldots K_{6}\right]$, where $K_{i}$ represents the cluster ID of the $i-t h$ row of $X_{E}$. We then run a second round of k-means clustering on the vector $\left[K_{1} \ldots K_{6}\right]$ of 55 patients, and transform the output centroids from the $K C$ form back into the $6 \times 8$ probability matrix form. We apply the same two-round k-means clustering process to $X_{C}$.

Using two-round clustering approach, we identify three centroids of $X_{E}$ and three centroids of $X_{C}$. Due to space constraint, the centroid matrices are not presented in this paper. Section II-B explains the interpretations of the different Eat and Correct clusters, and Figures 2 and 4 illustrate the results.

\section{B. Individualized Physiological Modeling}

We use a commonly accepted first-principle based compartmental physiological model proposed in [23] and refer to it as the Bergman model. The Bergman model we use is a firstorder differential equation that describes the interaction between the plasma insulin level and glucose level. To model the real-life scenario where a patient eats and takes subcutaneous insulin inputs, the Bergman model can be augmented with a second-order meal pathway that relates ingested carbohydrates to plasma glucose appearance [24], and a second-order subcutaneous insulin pathway that relates subcutaneous insulin inputs to plasma insulin appearance [25], [26]. The equations of the complete fifth-order augmented Bergman model are summarized in $[27]^{6}$. The model contains several physiological parameters that are patient specific: for example, the insulin sensitivity and basal glucose production rate. We tune the augmented Bergman model parameters within the value ranges reported in the original Bergman article [23], which include the glucose distribution rate, insulin sensitivity, meal glucose rate of appearance, and basal glucose production rate. For the other model parameters in the meal and insulin pathways, we use the nominal parameter values reported in the physiological modeling literature [26], [24].

\footnotetext{
${ }^{6} \mathrm{The}$ paper is available at http://repository.upenn.edu/cis_papers/782/
}

For each patient in the CSII dataset, we feed the recorded insulin and carbohydrate inputs to the augmented Bergman model and compare the model-generated glucose outputs with those in the CSII dataset. For each patient, we search for the combination of the tuning parameters on which the model achieves the best of fit on glucose outputs. Table $\mathrm{V}$ presents the key glucose statistics of the CSII measurement data and the simulated glucose outputs generated by the individualized model given the same insulin \& meal inputs. The individualized physiological model is able to reproduce the population statistics, such as the mean, maximum, and minimum glucose values, with errors that are not significant in the glucose control application (blood glucose meters have a typical measurement error ranging from 15 to $30 \mathrm{mg} / \mathrm{dL}$; the error range of CGM sensors is even larger). We also compare the glucose in and out of range percentages, using commonly accepted hypoglycemia threshold $70 \mathrm{mg} / \mathrm{dL}$ and hyperglycemia threshold $180 \mathrm{mg} / \mathrm{dL}$ [18]. Those are percentages of the glucose readings that are lower than 70 $\mathrm{mg} / \mathrm{dL}$, within $[70,180] \mathrm{mg} / \mathrm{dL}$, and higher than $180 \mathrm{mg} / \mathrm{dL}$. They are important clinical metrics of evaluating the risk of hypoglycemia and hyperglycemia, and are critical indicators of the quality of glycemic control. Table $\mathrm{V}$ shows that the individualized physiological model is able to reproduce the glucose in and out of range percentages observed in the CSII data. Table VI presents the per-patient differences of the statistics of the model-simulated glucose values and the real glucose measurements in the CSII dataset. It shows that the model is able to reproduce the key glucose statistics not only at the population level but also on each individual patient. We use the individually parameterized augmented Bergman model in the in silico closed-loop experiments.

\section{Closed-loop Experiments}

As stated in Section II-C, in order to demonstrate the utility of the proposed ETC modeling framework, we perform in silico experiments to show the effects of patient behavior (ETC type) change on the glycemic control outcomes. We build a model of the closed-loop system (see Figure 1) by integrating the ETC behavior model, the individualized physiological model, and a model of the BWZ. We use the PRISM probabilistic model checker [28] to evaluate the hypoglycemia and hyperglycemia rate of different instantiations of the system model: each instantiation is a specific pair of a user behavior model and an individualized physiological model. PRISM is an open-source tool for formal modeling and analysis of systems that exhibit probabilistic behavior. It can express and analyze several types of probabilistic models, such as discrete-time Markov chains, continuous-time Markov chains, and Markov decision processes, etc. We build the patient ETC behavior model as a discrete-time Markov chain, using probabilities derived from the CSII dataset as described in Section III-A. We also use Matlab to generate finite traces from the simulation of the individualized augmented Bergman model and encode them in PRISM. Then, we can use PRISM to exhaustively check every possible execution trace of the closed-loop system 
and compute the expected hypoglycemia and hyperglycemia rate.

Given a patient's individualized physiological model, we can pair it with his/her actual ETC type model or any other ETC types (there are 36 possible combinations) and run the model checking to evaluate the expected control outcomes. The in silico experimental results (e.g., those reported in Table II and Table III) identify the behavior change that might help a particular patient improve the glucose control outcomes, i.e., reducing the hypoglycemia and/or hyperglycemia rate.

\section{CONCLUSION}

In this paper, we propose the "Eat, Trust, and Correct" (ETC) framework for modeling T1D insulin pump user behavior. We use clustering algorithms to learn ETC user behavior patterns from a clinical dataset, and we tune a physiological model to fit each patient's clinical data. By running closedloop evaluation of the user behavior model and the individualized physiological model on a probabilistic model checker, we demonstrate that the ETC framework not only provides fresh clinical insights from the data but also enables exploring how switching behavior types may impact a patient's physiology. The outcomes of the closed-loop evaluation can inform T1D patient education and peer-support to improve the quality of glycemic control.

Our future work includes further validation of the ETC model by expanding the CSII dataset, exploring short-term Trust pattern fluctuations, and comparing different clustering techniques in the ETC framework.

\section{REFERENCES}

[1] C. for Disease Control, Prevention et al., "National diabetes statistics report: estimates of diabetes and its burden in the united states, 2014," Atlanta, GA: US Department of Health and Human Services, 2014.

[2] D. Bhatt and L. R. Reynolds, "Keep your hands off my insulin pump! the dilemma of the hospitalized insulin pump patient," The American journal of medicine, 2015.

[3] M. L. Misso, K. J. Egberts, M. Page, D. O’Connor, and J. Shaw, "Continuous subcutaneous insulin infusion (csii) versus multiple insulin injections for type 1 diabetes mellitus," The Cochrane Library, 2010.

[4] R. M. Bergenstal, W. V. Tamborlane, A. Ahmann, J. B. Buse, G. Dailey, S. N. Davis, C. Joyce, B. A. Perkins, J. B. Welsh, S. M. Willi et al., "Sensor-augmented pump therapy for a1c reduction (star 3) study results from the 6-month continuation phase," Diabetes Care, vol. 34, no. 11, pp. 2403-2405, 2011.

[5] G. Grunberger, J. M. Abelseth, T. S. Bailey, B. W. Bode, Y. Handelsman, R. Hellman, L. Jovanovič, W. S. Lane, P. Raskin, W. V. Tamborlane et al., "Consensus statement by the american association of clinical endocrinologists/american college of endocrinology insulin pump management task force," Endocrine Practice, vol. 20, no. 5, pp. 463-489, 2014.

[6] K. Jeitler, K. Horvath, A. Berghold, T. Gratzer, K. Neeser, T. Pieber, and A. Siebenhofer, "Continuous subcutaneous insulin infusion versus multiple daily insulin injections in patients with diabetes mellitus: systematic review and meta-analysis," Diabetologia, vol. 51, no. 6, pp. 941-951, 2008.

[7] M. M. Fatourechi, Y. C. Kudva, M. H. Murad, M. B. Elamin, C. C. Tabini, and V. M. Montori, "Hypoglycemia with intensive insulin therapy: a systematic review and meta-analyses of randomized trials of continuous subcutaneous insulin infusion versus multiple daily injections," The Journal of Clinical Endocrinology \& Metabolism, vol. 94, no. 3, pp. 729-740, 2009.
[8] J. Pickup and A. Sutton, "Severe hypoglycaemia and glycaemic control in Type 1 diabetes: meta-analysis of multiple daily insulin injections compared with continuous subcutaneous insulin infusion," Diabetic Medicine, vol. 25, no. 7, pp. 765-774, 2008.

[9] M. Abramowicz, G. Zuccotti, and J.-M. Pflomm, "Minimed 530g: An insulin pump with low-glucose suspend automation," pp. 1568-1568, 2015.

[10] J. Meece, "The artificial pancreas where we are, where were going," $A A D E$ in Practice, vol. 3, no. 2, pp. 42-44, 2015.

[11] S. D. Patek, S. Chen, P. Keith-Hynes, and I. Lee, "Distributed aspects of the artificial pancreas," in Communication, Control, and Computing (Allerton), 2013 51st Annual Allerton Conference on. IEEE, 2013, pp. 543-550.

[12] D. Sadigh, K. Driggs-Campbell, A. Puggelli, W. Li, V. Shia, R. Bajcsy, A. L. Sangiovanni-Vincentelli, S. S. Sastry, and S. A. Seshia, "Datadriven probabilistic modeling and verification of human driver behavior," Formal Verification and Modeling in Human-Machine Systems, 2014.

[13] N. Ahmed, E. de Visser, T. Shaw, A. Mohamed-Ameen, M. Campbell, and R. Parasuraman, "Statistical modelling of networked humanautomation performance using working memory capacity," Ergonomics, vol. 57, no. 3, pp. 295-318, 2014.

[14] M. Webster, C. Dixon, M. Fisher, M. Salem, J. Saunders, K. Koay, K. Dautenhahn, and J. Saez-Pons, "Toward reliable autonomous robotic assistants through formal verification: A case study," Human-Machine Systems, IEEE Transactions on, vol. PP, no. 99, pp. 1-11, 2015.

[15] R. W. Beck, W. V. Tamborlane, R. M. Bergenstal, K. M. Miller, S. N. DuBose, and C. A. Hall, "The t1d exchange clinic registry," The Journal of Clinical Endocrinology \& Metabolism, vol. 97, no. 12, pp. 43834389, 2012.

[16] A. D. Association et al., "Hypoglycemia (low blood glucose)," URL http://www. diabetes.org/living-with-diabetes/treatment-and-care/bloodglucose-control/hypoglycemia-low-blood. html, 2011.

[17] M. Heisler, S. Vijan, F. Makki, and J. D. Piette, "Diabetes control with reciprocal peer support versus nurse care management: a randomized trial," Annals of internal medicine, vol. 153, no. 8, pp. 507-515, 2010.

[18] R. L. Weinstein, S. L. Schwartz, R. L. Brazg, J. R. Bugler, T. A. Peyser, and G. V. McGarraugh, "Accuracy of the 5-day freestyle navigator continuous glucose monitoring system comparison with frequent laboratory reference measurements," Diabetes Care, vol. 30, no. 5, pp. 1125-1130, 2007.

[19] H.-P. Kriegel, P. Kröger, and A. Zimek, "Clustering high-dimensional data: A survey on subspace clustering, pattern-based clustering, and correlation clustering," ACM Transactions on Knowledge Discovery from Data (TKDD), vol. 3, no. 1, p. 1, 2009.

[20] R. Agrawal, J. Gehrke, D. Gunopulos, and P. Raghavan, Automatic subspace clustering of high dimensional data for data mining applications. ACM, 1998, vol. 27, no. 2.

[21] J. A. Hartigan and M. A. Wong, "Algorithm as 136: A k-means clustering algorithm," Applied statistics, pp. 100-108, 1979.

[22] B. A. Kohl, S. Chen, M. Mullen-Fortino, and I. Lee, "Evaluation and enhancement of an intraoperative insulin infusion protocol via in-silico simulation," in Healthcare Informatics (ICHI), 2013 IEEE International Conference on. IEEE, 2013, pp. 307-316.

[23] R. N. Bergman, Y. Z. Ider, C. R. Bowden, and C. Cobelli, "Quantitative estimation of insulin sensitivity." American Journal of PhysiologyEndocrinology And Metabolism, vol. 236, no. 6, p. E667, 1979.

[24] R. Gillis, C. C. Palerm, H. Zisser, L. Jovanovic, D. E. Seborg, and F. J. Doyle, "Glucose estimation and prediction through meal responses using ambulatory subject data for advisory mode model predictive control," Journal of diabetes science and technology, vol. 1, no. 6, pp. 825-833, 2007.

[25] T. Kobayashi, S. Sawano, T. Itoh, K. Kosaka, H. Hirayama, and Y. Kasuya, "The pharmacokinetics of insulin after continuous subcutaneous infusion or bolus subcutaneous injection in diabetic patients," Diabetes, vol. 32, no. 4, pp. 331-336, 1983.

[26] G. Nucci and C. Cobelli, "Models of subcutaneous insulin kinetics. a critical review," Computer methods and programs in biomedicine, vol. 62, no. 3, pp. 249-257, 2000.

[27] S. Chen, J. Weimer, M. Rickels, A. Peleckis, and I. Lee, "Towards a model-based meal detector for type I diabetics," in Medical CyberPhysical Systems Workshop 2015, 2015.

[28] M. Kwiatkowska, G. Norman, and D. Parker, "PRISM 4.0: Verification of probabilistic real-time systems," in CAV. Springer, 2011, pp. 585591. 